\title{
El compromiso y entrega en el aprendizaje, la competencia motriz percibida y la ansiedad ante el error y situaciones de estrés: factores de motivación de logro durante las clases de educación física en adolescentes
}

\author{
Márquez-Barquero, Magally; Azofeifa-Mora, Christian \\ El compromiso y entrega en el aprendizaje, la competencia motriz percibida y la ansiedad ante el error y \\ situaciones de estrés: factores de motivación de logro durante las clases de educación física en adolescentes \\ MHSalud, vol. 16, núm. 1, 2019 \\ Universidad Nacional, Costa Rica \\ Disponible en: http://www.redalyc.org/articulo.oa?id=237057357003 \\ DOI: https://doi.org/10.15359/mhs.16-1.3
}

Esta obra está bajo una Licencia Creative Commons Atribución-NoComercial-SinDerivar 3.0 Internacional. 


\title{
El compromiso y entrega en el aprendizaje, la competencia motriz percibida y la ansiedad ante el error y situaciones de estrés: factores de motivación de logro durante las clases de educación física en adolescentes
}

\author{
Learning Delivery and Commitment, Perceived Motor Competence, and Anxiety When Making Mistakes or \\ Under Stress: Motivating Factors in Adolescents for Achievement During Physical Education Classes
}

Comprometimento e entrega na aprendizagem, percepção de competência e ansiedade de condução frente aos erros e situações de estresse: fatores motivacionais nos adolescentes durante as aulas de educação física

Magally Márquez-Barquero

Universidad Nacional, Costa Rica

magally.marquez.barquero@una.cr

Christian Azofeifa-Mora

Universidad Nacional, Costa Rica

cristian.azofeifa.mora@una.cr
DOI: https://doi.org/10.15359/mhs.16-1.3

Redalyc: http://www.redalyc.org/articulo.oa? id $=237057357003$

\author{
Recepción: 18 Abril 2018 \\ Aprobación: 20 Septiembre 2018
}

\section{Resumen:}

Objetivo: determinar los factores asociados a la motivación de logro en los estudiantes de III Ciclo (séptimo, octavo y noveno) de la Educación General Básica durante las clases de Educación Física. Metodología: Se aplicó el cuestionario AMPET (Achievement Motivation in Physical Education Test) a un total de 396 estudiantes (178 mujeres y 218 hombres) de secundaria en cinco centros educativos públicos ubicados en el cantón Central de la Provincia de Heredia, Costa Rica. Se realizó análisis descriptivo promedios y desviaciones estándar, ANOVA factorial $2 \times 3$ para grupos independientes. Resultados: Se observan diferencias ( $\mathrm{p}$ $=.000)$ en el factor de compromiso y entrega en el aprendizaje según el nivel académico. Al realizar un post-hoc de Tukey, se encontró que a menor nivel académico mayor es el compromiso y entrega en el aprendizaje; es decir, los estudiantes de séptimo grado reflejan un mayor puntaje en el factor de compromiso y entrega en el aprendizaje (4.03 .06$)$ en comparación con los

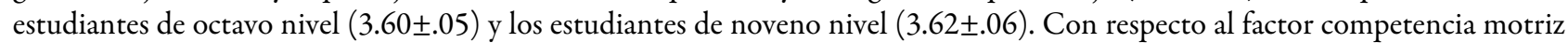
percibida, se obtuvieron diferencias $(\mathrm{p}=.000)$ según el sexo. Lo anterior refleja que los hombres, poseen una mayor competencia

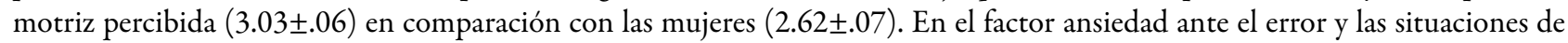
estrés, se encontraron diferencias estadísticamente significativas $(\mathrm{p}=.000)$ según el sexo, las mujeres obtuvieron un puntaje mayor,

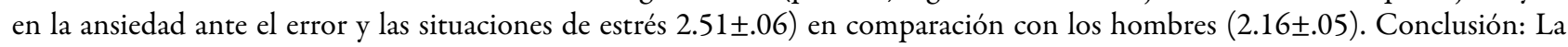
investigación reveló que las mujeres responden diferente que los hombres a los factores asociados a la motivación de logro y cabe resaltar que EF es una fuente de estrés para ellas, dato importante de analizar para identificar a qué obedece y determinar si se debe a que existe un desfase en la competencia motriz, lo que acarrea consecuencias en el comportamiento y comparar si dicho comportamiento se repite en los centros educativos de las distintas regiones del país.

Palabras Clave: motivación, educación física, adolescencia.

\section{Abstract:}

Objective: to determine the factors associated to motivation in students for achievement of III cycle (seventh, eighth, and ninth grades) of the General Basic Education during Physical Education classes. Methodology: The AMPET questionnaire (Achievement Motivation in Physical Education Test) was applied to a total of 396 students (178 women and 218 men) of high school from five public educational centers, located in the Central Canton of the Province of Heredia, Costa Rica. A descriptive analysis was made with averages and standard deviations, $2 \times 3$ factorial ANOVA for independent groups. Results: Differences $(\mathrm{p}=.000)$ were observed in the commitment factor and learning delivery according to the academic level. When doing a posthoc Tukey, it was found that the lower the academic level, the greater the commitment and learning delivery; that is to say, the seventh grade students reflect a higher score in the factor of commitment and learning delivery $(4.03 \pm .06)$ in comparison with eighth level $(3.60 \pm .05)$ and ninth level students $(3.62 \pm .06)$. With respect to the perceived motor competence factor, differences were obtained $(\mathrm{p}=.000)$ according to sex. The above reflects that men have a greater perceived motor competence $(3.03 \pm .06)$, compared to women $(2.62 \pm .07)$. Regarding the anxiety factor when making mistakes or under stress situations, statistically significant differences were found $(\mathrm{p}=.000)$; according to sex, women obtained a higher score in anxiety when making mistakes or under stress situations $2.51 \pm .06)$, compared to men $(2.16 \pm .05)$. Conclusion: The research revealed that women respond differently than men to the factors associated with achievement motivation and it should be noted that physical education is a 
source of stress for them, an important fact to analyze to know its cause, and whether it is due to the existence of a gap in motor competence that has consequences on behavior, as well as to compare whether this behavior is repeated in schools in different regions of the country.

KEYWORDS: motivation, physical education, adolescence.

\section{Resumo:}

Objetivo: determinar os fatores motivacionais de realização nos alunos do III ciclo (sétimo, oitavo e nono ano) da Educação Básica Geral durante as aulas de Educação Física. Metodologia: foi aplicado o questionário AMPET ( Achievement Motivation in Physical Education Test) ) a um total de 396 estudantes (178 mulheres e 218 homens) do ensino médio de cinco centros educacionais públicos localizados na região Central da Província de Heredia, Costa Rica. Foi realizada análise descritiva das médias e desvios-padrão, ANOVA fatorial $2 \times 3$ para grupos independentes. Resultados: se observam diferenças $(p=0,000)$ no fator de comprometimento e entrega na aprendizagem de acordo com o nível acadêmico. Ao fazer um Tukey post-hoc, verificou-se que quanto menor o nível acadêmico, maior o comprometimento e a entrega na aprendizagem; ou seja, os alunos da sétima série refletem mais pontos no fator de comprometimento e entrega na aprendizagem $(4,03 \pm 0,06)$ em comparação aos alunos da oitavo serie $(3,60 \pm 0,05)$ e da nona serie $(3,62 \pm 0,06)$. Com relação ao fator de competência motora percebido, foram obtidas diferenças ( $p$ $=0,000)$ de acordo com o sexo. Esse resultado indica que os homens possuem maior competência motora percebida $(3,03 \pm 0,06)$ em relação às mulheres $(2,62 \pm 0,07)$. No fator ansiedade diante do erro e as situações de estresse, foram encontradas diferenças estatisticamente significantes $(\mathrm{p}=0,000)$ de acordo com o sexo, as mulheres obtiveram mais pontos nas situações de ansiedade antes do erro e de estresse $2,51 \pm 0,06)$ em comparação aos homens $(2,16 \pm 0,05)$. Conclusão: a pesquisa revelou que as mulheres respondem diferentemente que os homens aos fatores motivacionais de realização e deve-se notar que a EF é uma fonte de estresse para elas. Um fator importante para analisar é a causa e se é devido à existência de uma lacuna na competência motora que tem consequências sobre o comportamento, e comparar se esse comportamento se repete nas escolas de diferentes regiões do país.

Palavras-Chave: motivação, educação física, adolescência.

\section{INTRODUCCIÓN}

La motivación en el contexto de la Educación Física (EF) es una línea de investigación que ha sido sometida a análisis (Kaplan, Katz y Flum, 2012; Naranjo, 2009; Turabik y Baskan, 2015), con el propósito de conocer los factores que motivan al estudiantado en las clases de EF, porque esta es una asignatura básica en la promoción de la actividad física en los centros educativos, debido a que fomenta hábitos de estilos de vida activos y eso influye para que la motivación sea analizada (Lonsdale, et al., 2015; Ruiz-Pérez, Moreno-Murcia, RamónOtero y Alias-García, 2015).

Las estrategias educativas basadas en la teoría de la motivación de logro y metas, resultan claves en el éxito académico y en la adherencia hacia determinadas asignaturas y contenidos para el estudiantado, tal teoría señala que dicha población, cuando maneja una mayor motivación para el logro, suele obtener un mejor rendimiento en el aprendizaje, en comparación con quienes se encuentran motivados a evitar el fracaso (Becerra-González y Reidl, 2015; Durán-Aponte y Elvira-Valdés, 2015; García, 2016; Huayllaquispe, 2013).

Dentro de esta teoría y metas se han generado distintas dimensiones para una compresión más profunda, en la cual aspectos relacionados con el compromiso hacia el aprendizaje, la competencia percibida y la ansiedad ante el error y situaciones de estrés, se enmarcan como factores que pueden ser considerados como elementos que expresen la motivación del estudiantado (Requena, 2014; Ruiz-Pérez, et al., 2015).

Si bien las motivaciones de los educandos hacia las clases de EF suelen ser variadas, identificar las tendencias hacia una motivación de logro o de evitar el fracaso, resulta esencial dentro del proceso del desarrollo de las competencias motrices del estudiantado y a la vez, permite identificar y relacionar motivaciones intrínsecas asociadas a la diversión, esfuerzo, y de forma negativa, hacia la ansiedad relacionada con el fracaso, que las clases de EF puedan generar en ellos (Carrasco, Parra, y Pérez, 2015; González, Cecchini, Llavona, y Amaro, 2010; Rubio-Castillo y Gómez-Mármol, 2016; Ruíz, Graupera, Contreras, y Nishida, 2004).

Al desempeñar tareas y otras acciones basados en una motivación de logro, la misma se ve influenciada por una serie de factores inherentes dentro de los que se destacan el compromiso y la entrega en el aprendizaje, 
que puede ser comprendido como aquel nivel con el cual los alumnos pueden estar implicados de forma activa para adquirir nuevo conocimiento y en donde se pone de manifiesto los altos niveles de intensidad y emotividad al emprender actividades de corte académico en buscar de nuevos conocimientos (Turabik \& Baskan, 2015; Yamila \& Donolo, 2014). Otro elemento influyente es la competencia motriz, percibida por los alumnos como la capacidad mediante la cual pueden actuar con eficiencia para desarrollar cada una de las tareas encomendadas en las clases de EF (López, Camerino, \& Castañer, 2015); un último componente a ser considerado, es la ansiedad detectada ante la posibilidad de error y el estrés generado por la posibilidad de fracaso durante las tareas en las clases de EF (Coterón, Álvarez, Pérez-Tejero, \& Sampedro, 2013; Lonsdale, et al., 2015)

La presencia de motivaciones hacia el logro en las clases de EF, ha sido investigada por Sánchez-Alcaraz, Gómez-Mármol y Más (2016), quienes analizaron sus dimensiones y las orientaciones y motivaciones en las clases de EF, al encontrar que los hombres perciben muestran una mayor competencia motriz, mientras que las mujeres una mayor ansiedad ante el fracaso y el estrés. Además, mostraron que las puntuaciones más bajas se relacionan con el compromiso y entrega hacia el aprendizaje, y puntuaciones altas están relacionados con la competencia motriz, las cuales se observaron en la población de forma general.

Coterón, Álvarez, Pérez-Tejero y Sampedro (2013), estudiaron factores asociados a la motivación de logro en estudiantes de secundaria durante las clases de EF, entre sus principales resultados reportaron que los estudiantes del sistema de enseñanza obligatoria secundaria percibían mayor ansiedad $(\mathrm{p}=.05)$ que los estudiantes de bachillerato; así mismo, según el tipo de centro educativo los estudiantes de centros públicos mostraron mayores niveles de compromiso $(\mathrm{p}=.05)$ y los de centros privados mostraron mayores valores de competencia percibida $(\mathrm{p}=.01)$ y ansiedad $(\mathrm{p}=.05)$. En lo que se refiere al sexo, se observó que los hombres presentan una mayor competencia motriz $(\mathrm{p}=.01)$ y compromiso $(\mathrm{p}=.01)$, mientras que las mujeres mostraron mayores niveles de ansiedad $(\mathrm{p}=.01)$.

Carrasco, et al., (2015) analizaron los factores asociados a la motivación de logro en las clases de EF y encontraron diferencias asociadas a la edad $(\mathrm{p}=.05)$ y la competencia motriz percibida, al evidenciar que a más edad mayor es su competencia motriz percibida y conforme la edad disminuye los valores relacionados con la ansiedad ante el error fueron mayores. Respecto al sexo, los hombres manisfestaron un mayor compromiso y entrega en el aprendizaje y una mayor competencia motriz percibida $(p=.001)$, mientras que las mujeres mostraron valores más altos en el factor ansiedad ante el error $(\mathrm{p}=.01)$ en comparación con los hombres.

Ante la necesidad de identificar los factores inherentes a la motivación de logro que prevalecen durante las clases de EF, el objetivo del estudio fue determinar el compromiso y entrega en el aprendizaje; la competencia motriz percibida y la ansiedad ante el error y situaciones de estrés del estudiantado de III Ciclo (séptimo, octavo y noveno) en centros educativos públicos ubicados en la Región Central de la provincia de Heredia.

\section{Metodología}

\section{Diseño}

La investigación se desarrolló como un estudio descriptivo longitudinal, la secuencia longitudinal, el control de los factores fue observacional, prospectivo. Las variables tratadas no se vieron manipuladas por la aplicación de un tratamiento determinado y el fin primordial fue describir el comportamiento de las variables comportamientos por medio de la aplicación de análisis de estadística descriptiva e inferencial, a través de la recolección de datos, con un instrumento, en el grupo de participantes. 


\section{Participantes}

La selección de la muestra se realizó con el método no probabilístico con sujetos voluntarios, se reclutó a 396 personas, provenientes de cinco centros educativos cuya distribución porcentual fue 38.9, 16.4, 13.6, 17.4 y 13.7, respectivamente. Previa a su participación en el estudio, cada individuo entregó el asentimiento y el consentimiento informado solicitados por el comité de ética. En la Tabla 1 se resumen las características de los participantes.

TABLA 1

Distribución de la cantidad de estudiantes según el sexo y nivel

\begin{tabular}{lllll}
\hline- & Hombres & & Mujeres \\
\hline Nivel & $\mathrm{n}$ & Edad(años) & $\mathrm{n}$ & Edad (años) \\
\cline { 2 - 5 } Séptimo & 57 & $12.9 \pm .74$ & 64 & $12.7 \pm .74$ \\
Octavo & 86 & $14.2 \pm .93$ & 70 & $13.7 \pm .91$ \\
Noveno & 75 & $14.9 \pm .78$ & 44 & $14.6 \pm .78$ \\
\hline
\end{tabular}

Fuente: Elaboración Propia

\section{Instrumentos}

Se utilizó el Test AMPET de motivación de logro para el aprendizaje en Educación Física (Achievement Motivation in Physical Education Test) (Ruiz, et al., 2004); que analiza los factores compromiso y entrega en el aprendizaje (.86); competencia motriz percibida (.82) y ansiedad ante el error en las situaciones de estrés (.87) y ha sido aplicado a poblaciones en edad escolar (López, Álvarez, Pérez-Tejero \& Molinuevo, 2013; Sánchez Alcaraz, Bernardino Ruiz-Pérez, Moreno-Murcia, Ramón-Otero \& Alias-García, 2015; Ruíz Pérez, 2014 y Sánchez Alcaraz, Bernardino Javier; Gómez Mármol, 2016); el cual cuenta con 37 ítems agrupados en tres dimensiones: compromiso y entrega en el aprendizaje ("Me concentro en lo que tengo que practicar durante la clase de educación física”); competencia motriz percibida ("Para las clases de educación física, pienso que poseo mejores capacidades que otros compañeros y compañeras") y ansiedad ante el error y situaciones de estrés ( "En la mayoría de las clases de educación física me pongo nervioso/a cuando tengo que realizar los ejercicios delante de mis compañeros/as del grupo y esto hace que no dé lo mejor de mí”). Las respuestas deben darse en una escala tipo Likert con cinco alternativas, desde (1) totalmente en desacuerdo, hasta (5) totalmente de acuerdo. La consistencia interna reportada fue: $\mathrm{a}=.87$ para compromiso y entrega con el aprendizaje, $\mathrm{a}=.82$ competencia motriz percibida y $\mathrm{a}=.84$ ansiedad ante el error y situaciones de estrés.

\section{Procedimientos}

Por las características de la población se estableció una logística de trabajo desarrollada en dos fases.

\section{Fase I}

Se visitó los centros educativos para coordinar con la administración los permisos de acceso y reclutamiento de los individuos y hacer la entrega de los consentimientos y los asentimientos informados. Se acordó el día y la fecha de la aplicación del instrumento con el profesional de educación física responsable del grupo de participantes. 


\section{Fase II}

Para la aplicación del cuestionario se contó con 7 personas colaboradoras, quienes reunieron a los participantes en el aula y una vez ahí, se les entregó un documento por persona, se procedió a leer las instrucciones y a evacuar dudas. Posteriormente cada participante contestó por escrito las preguntas de los enunciados del Test de AMPET.

\section{Análisis estadístico}

Se realizó el análisis descriptivo promedios y desviaciones estándar, así como ANOVA factorial 2x3, para grupos independientes post hoc de Tukey para analizar las interacciones entre sexos. El nivel de significancia establecido fue $\mathrm{p}<.05$. Se empleó el paquete estadístico IBM SPSS Statistics $\odot 24$ versión para Windows.

\section{Resultados}

TABLA 2

Valores promedios y desviaciones estándar relacionados con el puntaje obtenido en cada factor asociado a la motivación de logro según el sexo y grado escolar

\begin{tabular}{|c|c|c|c|c|c|}
\hline actores & \multicolumn{2}{|l|}{ Sexo } & \multicolumn{3}{|c|}{ Grado escolar } \\
\hline \multirow{2}{*}{$\begin{array}{l}\text { Factores } \\
\text { Compromiso } \\
\text { y entrega en } \\
\text { el } \\
\text { aprendizaje }\end{array}$} & Hombres & Mujer & Séptimo & Octavo & Noveno \\
\hline & $\begin{array}{l}3.74 \pm \\
0.64\end{array}$ & $\begin{array}{l}3.73 \\
\pm \\
0.82\end{array}$ & $\begin{array}{l}4.03 \pm \\
0.62\end{array}$ & $\begin{array}{l}3.61 \pm \\
0.79\end{array}$ & $\begin{array}{l}3.74 \pm \\
0.72\end{array}$ \\
\hline $\begin{array}{l}\text { Competencia } \\
\text { motriz } \\
\text { percibida }\end{array}$ & $\begin{array}{l}3.04 \pm \\
0.94\end{array}$ & $\begin{array}{l}2.62 \\
\pm \\
0.85\end{array}$ & $\begin{array}{l}2.8 \pm \\
0.8\end{array}$ & $\begin{array}{l}2.86 \pm \\
1\end{array}$ & $\begin{array}{l}2.85 \pm \\
.93\end{array}$ \\
\hline $\begin{array}{l}\text { Ansiedad } \\
\text { ante el error y } \\
\text { las } \\
\text { situaciones } \\
\text { de estrés }\end{array}$ & $\begin{array}{l}2.16 \pm \\
0.73\end{array}$ & $\begin{array}{l}2.53 \\
\pm \\
0.87\end{array}$ & $\begin{array}{l}2.29 \pm \\
0.81\end{array}$ & $\begin{array}{l}2.38 \pm \\
.84\end{array}$ & $\begin{array}{l}2.33 \pm \\
.81\end{array}$ \\
\hline
\end{tabular}

Fuente: Elaboración Propia 
TABLA 3

Resultados del análisis de ANOVA factorial para cada uno de los factores asociados a la motivación de logro en función del grado escolar y el sexo

\begin{tabular}{|c|c|c|c|c|c|c|}
\hline & \multicolumn{2}{|l|}{ Sexo } & \multicolumn{2}{|c|}{ Grado escolar } & \multicolumn{2}{|c|}{$\begin{array}{l}\text { Interacción } \\
\text { sexo x grado } \\
\text { escolar }\end{array}$} \\
\hline Factor & $\mathrm{F}$ & Sig. & $F$ & Sig. & $\mathrm{F}$ & sig. \\
\hline $\begin{array}{l}\text { Compromiso } \\
\text { y entrega en } \\
\text { el } \\
\text { aprendizaje }\end{array}$ & .243 & .623 & 14.496 & $.000^{*}$ & 1.095 & .335 \\
\hline $\begin{array}{l}\text { Competencia } \\
\text { motriz } \\
\text { percibida } \\
\text { Ansiedad } \\
\text { ante el error }\end{array}$ & 18.736 & $.000^{*}$ & .025 & .975 & 2.079 & .126 \\
\hline $\begin{array}{l}\text { y las } \\
\text { Situaciones } \\
\text { de estrés }\end{array}$ & 18.600 & $.000^{*}$ & .949 & .388 & .732 & .482 \\
\hline
\end{tabular}

${ }^{*} p<.05$ Fuente: Elaboración Propia

Por medio del análisis de ANOVA factorial, se observaron diferencias significativas (Tabla 3 ) en el puntaje promedio obtenido (Tabla 2) para el factor "compromiso y entrega en el aprendizaje”, según el grado escolar. Dichas diferencias, se encontraron por medio de un análisis post hoc de Tukey (Tabla 4), entre el puntaje obtenido por los estudiantes de séptimo año con respecto a los niveles de octavo y noveno; lo que muestra una disminución en la injerencia del compromiso y la entrega en el aprendizaje para incentivar la motivación de logro en las clases de educación física conforme el nivel académico aumenta para ambos sexos (Figura 1).

Con respecto a los factores "competencia motriz percibida" y "ansiedad ante el error y las situaciones de estrés", no se perciben diferencias significativas (Tabla 3), en sus puntuaciones según el grado escolar; sin embargo, parecen mostrar un comportamiento muy homogéneo en los distintos grados escolares (Figura 2 y Figura 3).

En lo relacionado al sexo, se advierten diferencias estadísticamente significativas (Tabla 3) para los factores "competencia motriz percibida" y "ansiedad ante el error y las situaciones de estrés"; en el factor de "competencia motriz percibida", se observa un puntaje promedio mayor (Tabla 2) en los hombres con respecto a las mujeres, lo que muestra, de forma significativa, una influencia mayor de este como elemento propiciador en la motivación hacia el logro durante las clases de EF.

Para el elemento "ansiedad ante el error y las situaciones de estrés", también se muestran diferencias significativas en los puntajes promedios entre sexos (Tabla 2). En este caso, las mujeres presentan valores más altos que los hombres lo cual indica que en las mujeres este factor es negativo para la motivación de logro, en las clases de EF.

Por su parte, el factor "compromiso y entrega en el aprendizaje", no muestra diferencias significativas (Tabla 3), en el puntaje promedio entre sexos (Tabla 2), lo que evidencia un comportamiento muy similar entre ambos sexos.

En la relación entre sexo y grado escolar, no se observó ninguna interacción significativa, para los componentes en estudio, relacionados con la motivación de logro. Si bien, los datos muestran en el factor "compromiso y entrega en el aprendizaje" una tendencia en el puntaje hacia la disminución, conforme se incrementa el nivel de secundaria, dichos datos no constituyen un valor significativo (Figura 1).

Con base en la interacción entre las variables y el factor "competencia motriz percibida", en el caso de los hombres, parece mostrar una tendencia al incremento en los puntajes conforme aumenta el grado escolar, mientras que en las mujeres dichos puntajes presentan una disminución cuando aumenta el grado escolar 
(Figura 2); no obstante, dichos resultados no representan una interacción significativa. Por su parte, el elemento "ansiedad ante el error y las situaciones de estrés", mostró un incremento en los puntajes en ambos sexos conforme el grado escolar aumenta, al revelar que los cambios más acentuados se presentan en las mujeres en asociación con el grado escolar (Figura 3), pero los resultados obtenidos no arrojaron interacciones significativas.

TABLA 4

Resultados del análisis de pruebas post hoc para las comparaciones múltiples en cada uno de los factores asociados a la motivación de logro en función del nivel de secundaria cursado

\begin{tabular}{|c|c|c|c|c|c|c|c|}
\hline - & - & $\begin{array}{l}\text { Compromis } \\
\text { entrega en } \\
\text { aprendizaje }\end{array}$ & & $\begin{array}{l}\text { Competencia } \\
\text { motriz perit }\end{array}$ & & $\begin{array}{l}\text { Ansiedad an } \\
\text { error y las } \\
\text { situaciones } \\
\text { estrés }\end{array}$ & de el \\
\hline $\begin{array}{l}\text { Nivel de } \\
\text { secundar }\end{array}$ & & $\begin{array}{l}\text { Diferencias } \\
\text { entre las } \\
\text { medias }\end{array}$ & Sig. & $\begin{array}{l}\text { Diferencias } \\
\text { entre las } \\
\text { medias }\end{array}$ & sig. & $\begin{array}{l}\text { Diferencias } \\
\text { entre las } \\
\text { medias }\end{array}$ & Sig. \\
\hline Séptimo & $\begin{array}{l}\text { Octavo } \\
\text { Noveno }\end{array}$ & $\begin{array}{l}.4152^{*} \\
4166^{*}\end{array}$ & $\begin{array}{l}.000 \\
.000\end{array}$ & $\begin{array}{l}-.0528 \\
-.0980\end{array}$ & $\begin{array}{l}.881 \\
.682\end{array}$ & $\begin{array}{l}-.0950 \\
-.0010\end{array}$ & $\begin{array}{l}.591 \\
1.000\end{array}$ \\
\hline Octavo & $\begin{array}{l}\text { Séptimo } \\
\text { Noveno }\end{array}$ & $\begin{array}{l}-.4152^{*} \\
.0014\end{array}$ & $\begin{array}{l}.000 \\
1.000\end{array}$ & $\begin{array}{l}.0528 \\
-.0452\end{array}$ & $\begin{array}{l}.881 \\
.912\end{array}$ & $\begin{array}{l}.0950 \\
.0940\end{array}$ & $\begin{array}{l}.591 \\
.601\end{array}$ \\
\hline Noveno & $\begin{array}{l}\text { Séptimo } \\
\text { octavo }\end{array}$ & $\begin{array}{l}-.4166^{*} \\
-.0014\end{array}$ & $\begin{array}{l}.000 \\
1.000\end{array}$ & $\begin{array}{l}.0980 \\
.0452\end{array}$ & $\begin{array}{l}.682 \\
.912\end{array}$ & $\begin{array}{l}.0010 \\
-.0940\end{array}$ & $\begin{array}{l}1.000 \\
.601\end{array}$ \\
\hline
\end{tabular}

${ }^{*} \mathrm{p}<.05$ Fuente: Elaboración Propia

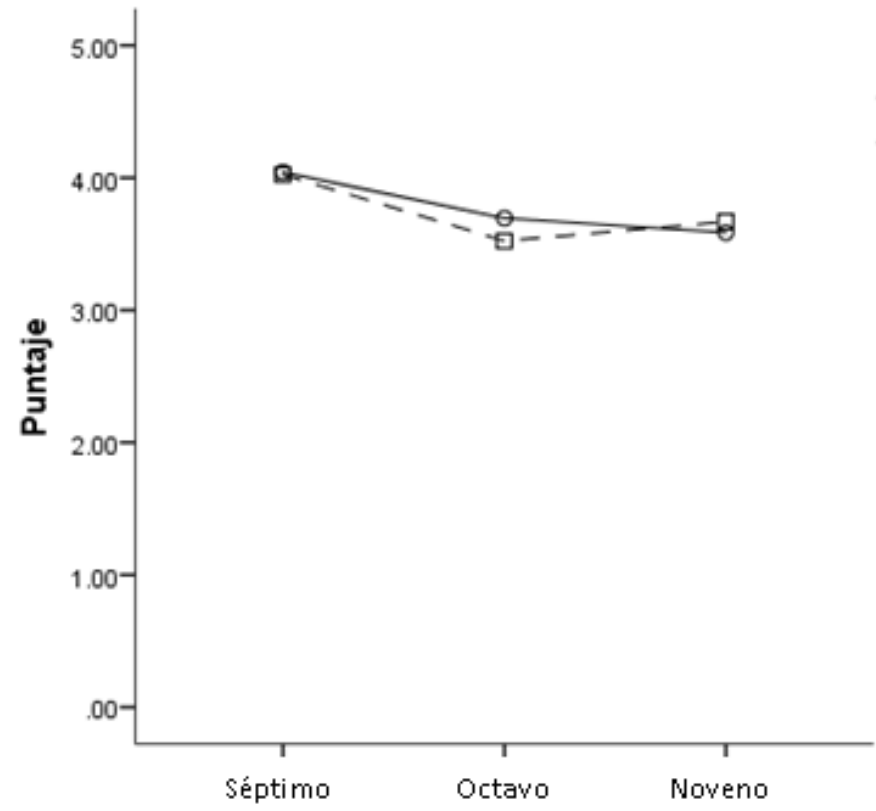

Compromiso y entrega en el aprendizaje

FIGURA 1

Valores de interacción del factor compromiso y entrega en el aprendizaje para la motivación de logro en las clases de educación física en función del sexo y nivel educativo.

Fuente: Elaboración Propia 


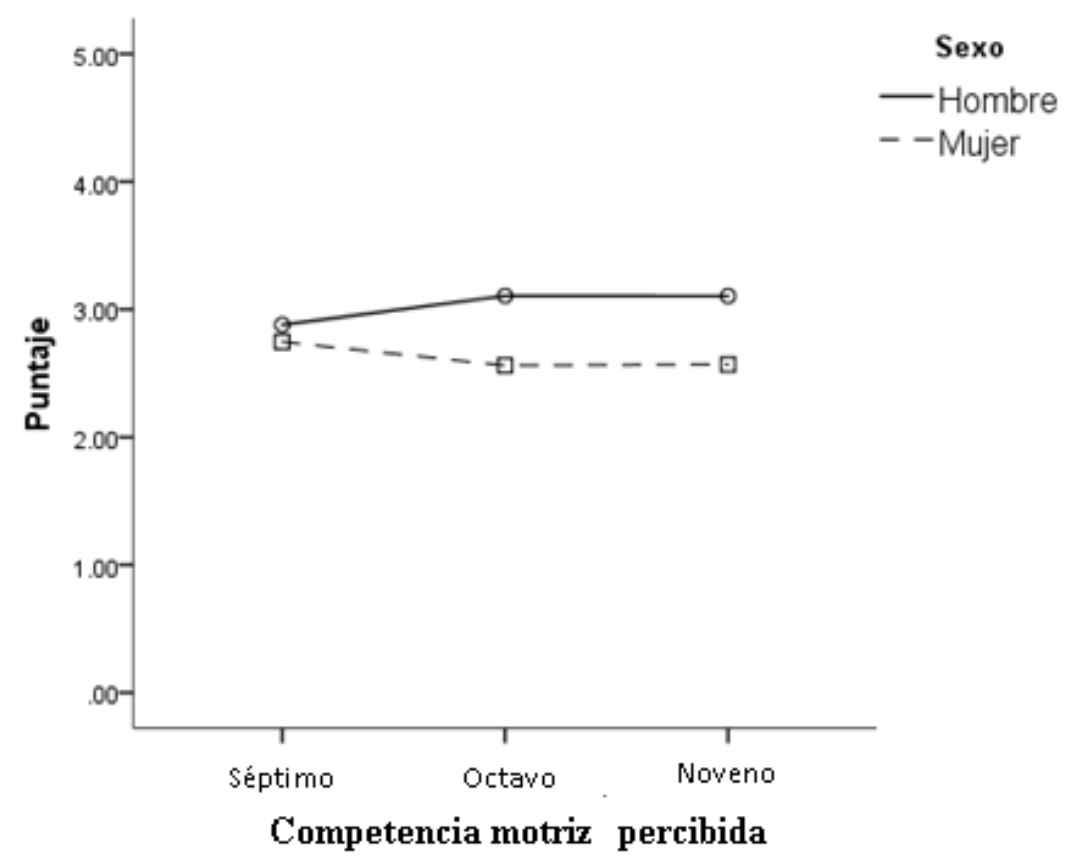

FIGURA 2

Valores de interacción del factor competencia motriz percibida para la motivación de logro en las clases de educación física en función del sexo y nivel educativo.

Fuente: Elaboración Propia

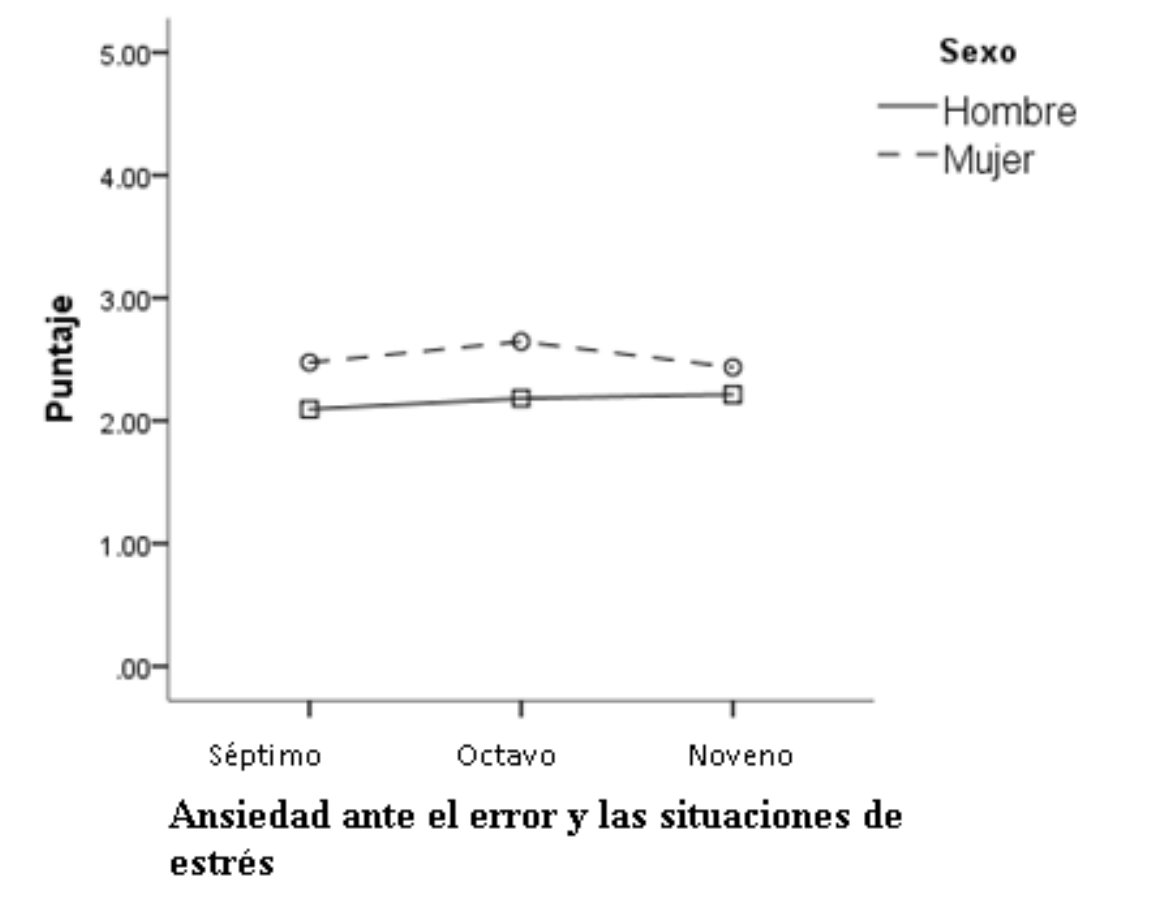

FIGURA 3

Valores de interacción del factor ansiedad ante el error y situaciones de estrés para la motivación de logro en las clases de educación física en función del sexo y nivel educativo. 


\section{Discusión}

Un conocimiento más profundo de los factores asociados a la motivación de logro en el estudiantado, puede incentivar su participación dentro de las clases de EF, lo que resulta ser un insumo valioso para la aplicación de estrategias pedagógicas acordes a la diversidad estudiantil actual (Lonsdale, et al., 2015; Ruiz-Pérez, et al., 2015); por lo anterior, este estudio analizó los elementos asociados a la motivación de logro en los estudiantes de III Ciclo de la Educación General Básica durante las clases de EF.

Se encontró, que el factor de compromiso y entrega en el aprendizaje depende del grado escolar en curso, lo que implica que a mayor grado escolar las estrategias para lograr que el estudiantado se interese en aprender, se deben adecuar a las características propias del desarrollo físico y mental y según Carrasco, et al., (2015) la pérdida de interés se ve reflejado en un cese en la mejora de las destrezas o bien, el no acatamiento de las instrucciones durante las clases de EF; por el contrario se observó una mayor orientación hacia el compromiso y la tarea en estudiantes de menor grado escolar y conforme aumenta el mismo, el factor competencia motriz percibida tiene una mayor impacto en los educandos, asociado a cambios en los ritmos de madurez evolutiva, la exposición a tareas motrices y la influencia del entorno social (López, Camerino, \& Castañer, 2015; Sánchez-Alcaraz, et al., 2016).

La competencia motriz percibida por los hombres y las mujeres (Coterón, et al., 2013; Sánchez-Alcaraz, et al., 2016) puede verse influencia por una mayor o menor práctica de actividad física extraclase que parece tener injerencia positiva en la competencia motriz observada en los hombres y por el contrario, una afectación negativa en las mujeres por la pobre participación en las clases de EF durante estos grados escolares (Carrasco, et al., 2015; Hellín, Moreno, \& Rodríguez, 2006; López, et al., 2015).

En el factor de ansiedad ante el error y las situaciones de estrés, las diferencias de un mayor puntaje de las mujeres con respecto a los hombres, muestran una similitud con otros estudios realizados (Coterón, et al., 2013; López, et al., 2015; Sánchez-Alcaraz, et al., 2016) y tal fenómeno puede asociarse con un efecto del contexto sociocultural sobre las apreciaciones que tienen las mujeres hacia la actividad física y los juicios valorativos que propician un rechazo hacia la misma, lo que incide sobre la competencia motriz al reflejar un déficit de habilidades y destrezas relacionadas con la EF.

\section{ConCluSión}

Los factores como el compromiso y entrega; la competencia motriz percibida y la ansiedad ante el error y situaciones de estrés, inciden en la clase de EF, esto debido a que es una disciplina multifactorial y dada la importancia que tiene la motivación, es por lo tanto un tema que merece ser analizado desde diferentes perspectivas, para así brindar herramientas que le permitan al profesional en EF conocer cómo se perciben y cómo se comparan los educandos con sus pares; qué es lo que les motiva y qué les limita para aprender y participar, debido a que las bajas percepciones son señal de baja motivación, escaso compromiso y la posibilidad de generar antipatía hacia la materia. La investigación reveló que las mujeres responden diferente que los hombres a los factores asociados a la motivación de logro y cabe resaltar que EF es una fuente de estrés en ellas, dato importante de analizar para determinar a qué obedece y si se debe a que existe un desfase en la competencia motriz, lo que acarrea consecuencias en el comportamiento y comparar si dicho comportamiento se repite en los centros educativos de las distintas regiones del país.

\section{REFERENCIAS}

Becerra-González, C., \& Reidl, L. (2015). Motivación, autoeficacia, estilo atribucional y rendimiento escolar de estudiantes de bachillerato. Revista Electrónica de Investigación Educativa, 17(173), 79-93. Recuperada de http://redie.uabc.mx/vol17no3/contenido-becerra-reidl.html 
Carrasco, M., Parra, D., \& Pérez, C. (2015). La competencia motriz y su percepción en el alumnado de cuarto curso de Educación Secundaria y Bachillerato. Calidad de Vida y Salud, 8(2), 70-87. Recuperada de http:// revistacdvs.uflo.edu.ar/index.php/CdVUFLO/article/view/119/128

Coterón, J., Álvarez, E., Pérez-Tejero, J., \& Sampedro, J. (2013). Clima motivacional, competencia percibida, compromiso y ansiedad en Educación Física. Diferencias en función de la obligatoriedad de la enseñanza. Revista de Psicología del Deporte, 22(1), 151-157. Recuperada de http://www.redalyc.org/ pdf/2351/235127552045.pdf

Durán-Aponte, E., \& Elvira-Valdés, M. (2015). Patrones atribucionales y persistencia académica en estudiantes universitarios: validez de la Escala Atribucional de Motivación de Logro General (EAML-G). Revista Intercontinental de Psicología y Educación, 17(2), 201-221. Recuperada de http://www.redalyc.org/ pdf/802/80247939011.pdf

García, J. M. (2016). La motivación de logro mejora el rendimiento académico. Revista Electrónica de Investigación y Docencia Creativa, 5, 1-8. Recuperada de http://digibug.ugr.es/bitstream/10481/39336/1/5-1.pdf

González, C., Cecchini, J. A., Llavona, A., \& Amaro, V. (2010). Influencia del entorno social $y$ el clima motivacional en el autoconcepto de las futbolistas asturianas. Aula Abierta, 38(1), 25-36. Recuperada de https://www.researchgate.net/ publication/44163447_Influencia_del_entorno_social_y_clima_motivacional_en_el_autoconcepto_de_las_futbolistas_asturianas

Hellín, P., Moreno, J. A., \& Rodríguez, P. (2006). Relación de la competencia motriz percibida con la práctica físico-deportiva. Revista de Psicología del Deporte, 15(2), 219-231. Recuperada de http://www.rpd-online.com/ article/view/232/232

Huayllaquispe, T. (2013). Desempeño docente del practicante de Educación Física y la motivación de logro para la clase en alumnos del nivel secundaria de las instituciones educativas de Lima, asignadas a las Prácticas Pre Profesionales UNMSM, año 2013. Universidad Nacional de Educación Enrique Guzmán y Valle. Recuperada de http://repositorio.une.edu.pe/bitstream/handle/UNE/968/TM CE-Cd H82 2015.pdf?sequence=1

Kaplan, A., Katz, I., \& Flum, H. (2012). Motivation theory in educational practice: Knowledge claims, challenges, and future directions. In APA educational psychology handbook. Individual differences and cultural and contextual factors. (Vol. 2, pp. 165-194). Washington: American Psychological Association. https:// doi.org/10.1037/13274-007

Lonsdale, C., Lester, A., Owen, K. B., White, R. L., Moyes, I., Peralta, L., ... Lubans, D. R. (2015). An Internetsupported Physical Activity Intervention Delivered in Secondary Schools Located in Low Socio-economic Status Communities: Study Protocol for the Activity and Motivation in Physical Education (AMPED) Cluster Randomized Controlled Trial. BMC Public Health, 16(1), 17. https://doi.org/10.1186/s12889-015-2583-7

López, A., Camerino, O., \& Castañer, M. (2015). Evaluar la motivación en la educación física , una aplicación con AMPET*. Didáctica de la Educación Física, 47, 55-63. Retrieved from http://www.observesport.com/desktop/ images/docu/aj85rr9d.pdf

Naranjo, M. L. (2009). Motivación\#: Perspectivas teóricas y algunas consideraciones de su importancia en el ámbito educativo. Revista Educación, 33(2), 153-170. https://doi.org/http://dx.doi.org/10.15517/revedu.v33i2.510

Requena, C. M. (2014). Implicaciones de los estilos de enseñanza aprendizaje y de la educación emocional en la enseñanza de la danza. Universidad Nacional de Educación a Distancia. Recuperada de http://e-spacio.uned.es/ fez/eserv/tesisuned:Educacion-Crrequena/Documento.pdf

Rubio-Castillo, A. D., \& Gómez-Mármol, A. (2016, July 25). Efectos del Modelo Ludotécnico en el aprendizaje técnico, competencia y motivación en la enseñanza del baloncesto en Educación Física. Revista Euroamericana de Ciencias del Deporte, 5(2), 41-46. Recuperada de http://revistas.um.es/sportk/article/view/264631/196301

Ruiz-Pérez, L. M., Moreno-Murcia, J. A., Ramón-Otero, I., \& Alias-García, A. (2015). Motivación de logro para aprender en Educación Física: adaptación de la versión española del Test AM PET [ 1 ]. Revista Española de Pedagogía, (260), 157-176. Recuperada de http://content.ebscohost.com/ContentServer.asp? $\mathrm{T}=\mathrm{P} \& \mathrm{P}=\mathrm{AN} \& \mathrm{~K}=101375793 \& \mathrm{~S}=\mathrm{R} \& \mathrm{D}=\mathrm{ehh} \&$ EbscoContent $=\mathrm{dGJyMNLe} 80$ SeprU40dvuOLCmr0\%2BeqLFSsqy4SbW 
Ruíz, L., Graupera, J. L., Contreras, O., \& Nishida, T. (2004). Motivación de logro en educación física escolar: un estudio comparativo entre cinco países. Revista de Educación, 333, 345-361. Recuperada de http:// www.cafyd.com/educacionfinal.pdf

Sánchez-Alcaraz, B., Gómez-Mármol, A., \& Más, M. (2016). Estudio de la motivación de logro y orientación motivacional en estudiantes de educación física. Apunts. Educación Física y Deportes, (124), 35-40. https:// doi.org/10.5672/apunts.2014-0983.es.(2016/2).124.04

Turabik, T., \& Baskan, G. A. (2015). The importance of motivation theories in terms of education systems. ProcediaSocial and Behavioral Sciences, 186, 1055-1063. https://doi.org/10.1016/j.sbspro.2015.04.006

Yamila, D., \& Donolo, D. (2014). Factores clave en la promoción del compromiso en educación primaria. Un estudio sobre tasks, teachers and students. Congreso Iberoamericano de Ciencia, Tecnología, Innovación y Educación INTRODUCCIÓN, 1-17.

\section{BY-NC-ND}

